\title{
Face numbers of centrally symmetric polytopes produced from split graphs
}

\author{
Ragnar Freij \\ Dept. Mathematics and Systems Analysis \\ Aalto University \\ Espoo, Finland \\ ragnar.freij@aalto.fi \\ Moritz W. Schmitt** Günter M. Ziegler** \\ Institut für Mathematik \\ Freie Universität Berlin \\ Berlin, Germany \\ \{mws, ziegler\}@math.fu-berlin.de \\ Matthias Henze* \\ Institut für Informatik \\ Freie Universität Berlin \\ Berlin, Germany \\ matthias.henze@fu-berlin.de
}

Submitted: Feb 7, 2012; Accepted: May 6, 2013; Published: May 16, 2013

Mathematics Subject Classifications: 52B05, 52B12, 05C75

\begin{abstract}
We analyze a remarkable class of centrally symmetric polytopes, the Hansen polytopes of split graphs. We confirm Kalai's $3^{d}$ conjecture for such polytopes (they all have at least $3^{d}$ nonempty faces) and show that the Hanner polytopes among them (which have exactly $3^{d}$ nonempty faces) correspond to threshold graphs. Our study produces a new family of Hansen polytopes that have only $3^{d}+16$ nonempty faces.
\end{abstract}

Keywords: Hansen polytopes, Hanner polytopes, split graphs, threshold graphs, $3^{d}$ conjecture

\section{Introduction}

A convex polytope $P$ is centrally symmetric if $P=-P$. In 1989, Kalai [5] posed three conjectures on the number of faces and flags of centrally symmetric polytopes, which he

\footnotetext{
*Supported by Deutsche Forschungsgemeinschaft (DFG) within the project He 2272/4-1.

** Supported by DFG Research Center Matheon, Project F13, and by ERC Advanced Grant agreement no. 247029-SDModels.
} 
named Conjectures A, B and C. Two of these, Conjectures B and C, were refuted by Sanyal et al. [8]. The exposed counterexamples are so-called Hansen polytopes that are constructed from the stable set structure of certain self-complementary graphs. Conjecture A however, known as the $3^{d}$ conjecture, was confirmed for dimension $d \leqslant 4$ but remains open for $d>4$ :

Conjecture 1 ( $3^{d}$ conjecture). Every centrally symmetric polytope of dimension $d$ has at least $3^{d}$ nonempty faces.

The present article is a contribution to the quest for settling Kalai's conjecture. Based on the examples in [8], we have originally started this project by experimentally (via polymake [2]) searching for self-complementary graphs, which yield self-dual Hansen polytopes with interesting properties in view of the $3^{d}$ conjecture. It turned out, however, that (a) as expected, non-perfect graphs do not yield good results, and (b) the most interesting examples arise not from the "self-complementary/self-dual" criterion, but from certain split graphs, which are perfect but not self-complementary. This has led to the present study, which confirms this first experimental evidence.

As our main result, we express the number of nonempty faces of the Hansen polytopes of a split graph in terms of certain partitions of the node set of the underlying graph. In particular, we confirm the $3^{d}$ conjecture for such polytopes, and show that equality in this class corresponds to threshold graphs.

After introducing Hansen polytopes in Section 2, we analyze Hansen polytopes of threshold graphs, which are special split graphs, in Section 3. It turns out that a Hansen polytope is a Hanner polytope if and only if the underlying graph is threshold. In Section 4, we investigate the combinatorics of Hansen polytopes of general split graphs and prove the main result mentioned above. Our study moreover produces examples of centrally symmetric polytopes that have a total number of nonempty faces very close to the conjectured lower bound of $3^{d}$. The article closes in Section 5 with remarks on the behavior of our examples with respect to two conjectures that concern the minimal Mahler volume and the minimal number of full flags for a centrally symmetric $d$-polytope.

General assumptions. All our graphs are finite and simple. The set of nodes of a graph $G$ is denoted by $V(G)$, and similarly the edge set is $E(G)$ if no other notation is specified. The graph complement of $G$ is $\bar{G}$. The complete graph on $n$ nodes is $K_{n}$. All polytopes are convex. We denote the polar of a polytope $P$ by $P^{\star}$. For details on graph theory we refer to Diestel [1], for polytope theory to [10].

\section{Hansen Polytopes}

Hansen polytopes were introduced by Hansen [4] in 1977. One constructs them from the stable set structure of a (perfect) graph $G$ by applying the twisted prism operation to the stable set polytope. Let us define these terms.

Definition 2 (Twisted prism). Let $P \subset \mathbb{R}^{d}$ be a polytope and let $Q:=\{1\} \times P$ be its embedding in $\mathbb{R}^{d+1}$. The twisted prism of $P$ is defined as the convex hull of $Q$ and its reflected copy $-Q$, that is, $\operatorname{tp}(P):=\operatorname{conv}(Q \cup-Q)$. 
Twisted prisms are centrally symmetric by construction. We are interested in twisted prisms of stable set polytopes, which we introduce next (see also Schrijver [9, Sec. 64.4]). By $e_{i}$ we denote the $i$ th coordinate unit vector.

Definition 3. Let $G$ be a graph. The stable set polytope of $G$ is defined as

$$
\operatorname{stab}(G):=\operatorname{conv}\left\{\sum_{i \in I} e_{i}: I \subseteq V(G) \text { stable }\right\} .
$$

Now we can define the main object of our studies.

Definition 4 (Hansen polytope). The Hansen polytope of a graph $G$ is defined as the twisted prism of its stable set polytope, that is, $\mathrm{H}(G):=\operatorname{tp}(\operatorname{stab}(G))$.

Examples of Hansen polytopes are cubes (produced from empty graphs) and crosspolytopes (from complete graphs). Recall that a graph $G$ is perfect if the size of the largest clique of any induced subgraph $H$ of $G$ equals the chromatic number of $H$. The following properties of Hansen polytopes provide a link between its face structure and the combinatorics of the underlying graph.

Lemma 5. Let $G$ be a graph.

(i) The vertex set of $\mathrm{H}(G)$ is $\operatorname{vert}(\mathrm{H}(G))=\left\{ \pm\left(e_{0}+\sum_{i \in I} e_{i}\right): I \subseteq V(G)\right.$ stable $\}$.

(ii) If $G$ is perfect, then $\left\{-1 \leqslant-x_{0}+2 \sum_{i \in C} x_{i} \leqslant 1: C \subseteq V(G)\right.$ clique $\}$ is an irredundant facet description of $\mathrm{H}(G)$.

(iii) If $G$ is perfect, then the polar of the Hansen polytope of $G$ is affinely equivalent to the Hansen polytope of $\bar{G}$, in symbols $\mathrm{H}(G)^{\star} \cong \mathrm{H}(\bar{G})$.

Proof. Part (i) follows from the definition. A proof of (ii) can be found in Hansen's paper [4] and (iii) follows from (ii).

From now on for the rest of the article we assume all graphs to be perfect.

\section{Hansen Polytopes of Threshold Graphs}

An important class of polytopes that attain the conjectured lower bound of the $3^{d}$ conjecture are the so-called Hanner polytopes. This class of polytopes includes cubes and crosspolytopes and was introduced by Hanner [3] in 1956. They are recursively defined as follows.

Definition 6 (Hanner polytope). A polytope $P \subset \mathbb{R}^{d}$ is a Hanner polytope if it is either a centrally symmetric line segment or, for $d \geqslant 2$, the Cartesian product of two Hanner polytopes, or the polar of a Hanner polytope. 
It is neither the case that all Hanner polytopes are Hansen polytopes nor vice versa. A characterization of their relation is our first result. Before we can state it, we need to introduce threshold graphs, a subclass of perfect graphs. An extensive treatment of this graph class is Mahadev \& Peled [6]. The definition involves the notions of dominating and isolated nodes: A node in a graph is dominating if it is adjacent to all other nodes; it is isolated if it is not adjacent to any other node.

Definition 7 (Threshold graph). A graph $G$ is a threshold graph if it can be constructed from the empty graph by repeatedly adding either an isolated node or a dominating node.

This class of graphs is closed under taking complements.

Theorem 8. The Hansen polytope $\mathrm{H}(G)$ is a Hanner polytope if and only if $G$ is a threshold graph.

Proof. $(\Leftarrow)$ We use induction on the number of nodes of $G$. If $G$ is the empty graph, then $\mathrm{H}(G)$ is just a centrally symmetric segment, and therefore a Hanner polytope. Now assume that $G$ has $n+1$ nodes. Since the class of Hanner polytopes is closed under taking polars and $\mathrm{H}(G)^{\star} \cong \mathrm{H}(\bar{G})$, we can assume $G=T \bullet\{v\}$ with $T$ being threshold. Here $\bullet$ denotes the usual disjoint union of graphs and $v$ is a single node with $v \notin T$. The stable sets of $G$ are exactly the stable sets of $T$, with and without the new node $v$. Given a stable set $S$ of $T$ the vertices of $\mathrm{H}(G)$ are of the form $\pm\left(e_{0}+\sum_{i \in S} e_{i}\right)$ and $\pm\left(e_{0}+\sum_{i \in S} e_{i}+e_{n+1}\right)$, where we assign $v$ the label $n+1$. By the linear transformation defined by $e_{0} \mapsto e_{0}-e_{n+1}$, $e_{n+1} \mapsto 2 e_{n+1}$, and $e_{i} \mapsto e_{i}$ for $i=1, \ldots, n$, we get $\mathrm{H}(G)=\mathrm{H}(T \bullet v) \cong \mathrm{H}(T) \times[-1,1]$, which means that $\mathrm{H}(G)$ is a Hanner polytope, since $\mathrm{H}(T)$ is by hypothesis.

$(\Rightarrow)$ Assume that $\mathrm{H}(G)$ is Hanner. Again it is enough to cover just one case, namely $\mathrm{H}(G)=P \times P^{\prime}$ with $P, P^{\prime}$ being lower-dimensional Hanner polytopes. The stable set polytope $\operatorname{stab}(G)$ is a facet of $\mathrm{H}(G)$ and can therefore be written as $\operatorname{stab}(G)=Q \times Q^{\prime}$ with $Q, Q^{\prime}$ being faces of $P, P^{\prime}$, respectively. Since we have $\operatorname{dim}(Q)+\operatorname{dim}\left(Q^{\prime}\right)=\operatorname{dim}(\operatorname{stab}(G))=$ $\operatorname{dim}(P)+\operatorname{dim}\left(P^{\prime}\right)-1$, we can further assume that $Q=P$ and $Q^{\prime}$ is a facet of $P^{\prime}$. Let $q:=\operatorname{dim}(Q)$ and $q^{\prime}:=\operatorname{dim}\left(Q^{\prime}\right)$.

We now construct a threshold graph $H^{\prime}$ on $q^{\prime}$ nodes such that $G=\overline{K_{q}} \bullet H^{\prime}$. This will prove that $G$ is threshold as well. Since $\operatorname{stab}(G)$ is a product, we get $\operatorname{vert}(\operatorname{stab}(G))=$ $\operatorname{vert}(Q) \times \operatorname{vert}\left(Q^{\prime}\right)$. Each coordinate of a vertex of $\operatorname{stab}(G)$ corresponds to a node in $G$. Let $V_{1} \subseteq V(G)$ be the node set defined by the first $q$ coordinates and let $V_{2} \subseteq V(G)$ be the set defined by the last $q^{\prime}$ coordinates. Then

$$
\begin{aligned}
\operatorname{vert}(\operatorname{stab}(G))= & \left\{\sum_{i \in I} e_{i}: I \subseteq V(G) \text { stable set of } G\right\} \\
= & \left\{\sum_{i \in I} e_{i}: I \subseteq V_{1} \text { stable set of } G\left[V_{1}\right] \text { and } N(I) \cap V_{2}=\emptyset\right\} \\
& \times\left\{\sum_{i \in I} e_{i}: I \subseteq V_{2} \text { stable set of } G\left[V_{2}\right] \text { and } N(I) \cap V_{1}=\emptyset\right\},
\end{aligned}
$$

where $N(I)$ is the set of nodes adjacent to some node in $I$ and $G\left[V_{j}\right]$ is the subgraph of $G$ induced by $V_{j}, j=1,2$. In particular, we have $e_{i} \in \operatorname{vert}(\operatorname{stab}(G))$, for all $i=1, \ldots, q+q^{\prime}$. 
From this and the right-hand side of the equality above, we can deduce that there are no edges between $V_{1}$ and $V_{2}$. By setting $H^{\prime}:=G\left[V_{2}\right]$, we get $G=G\left[V_{1}\right] \cup H^{\prime}$. So what is left to show is that $G\left[V_{1}\right]$ is an edgeless graph and that $H^{\prime}$ is a threshold graph.

Let us first see that $H^{\prime}$ is threshold. Since $P \cong \operatorname{stab}\left(G\left[V_{1}\right]\right)$ is at least one-dimensional, $G\left[V_{1}\right]$ has one node minimum, i.e., $\left|V\left(H^{\prime}\right)\right|<|V(G)|$. From [3, Cor. $3.4 \&$ Thm. 7.4], we know that Hanner polytopes are twisted prisms over any of their facets, which means for us that $P^{\prime} \cong \operatorname{tp}\left(Q^{\prime}\right) \cong \mathrm{H}\left(H^{\prime}\right)$. Thus, by induction, $H^{\prime}$ is a threshold graph.

As $P \cong \operatorname{stab}\left(G\left[V_{1}\right]\right)$ is Hanner, it has a center of symmetry. So there exists a vector $c \in \mathbb{R}^{q}$ such that $\operatorname{stab}\left(G\left[V_{1}\right]\right)=-\operatorname{stab}\left(G\left[V_{1}\right]\right)+2 c$. The origin and all unit vectors $e_{i}$ for $1 \leqslant i \leqslant q$ are vertices of $\operatorname{stab}\left(G\left[V_{1}\right]\right)$, thus we must have $c=\left(\frac{1}{2}, \ldots, \frac{1}{2}\right)$. This means $\operatorname{stab}\left(G\left[V_{1}\right]\right)=[0,1]^{q}$, which in turn yields $G\left[V_{1}\right]=\overline{K_{q}}$.

Observe that not all Hanner polytopes can be represented as Hansen polytopes of perfect graphs. For example, the product of two octahedra is a Hanner polytope but not a Hansen polytope.

Since every Hanner polytope satisfies the $3^{d}$ conjecture with equality, we have the following

Corollary 9. If $G$ is a threshold graph, then $\mathrm{H}(G)$ satisfies the $3^{d}$ conjecture with equality.

\section{Hansen Polytopes of Split Graphs}

Now we analyze the Hansen polytopes of split graphs. It is easy to verify and well-known that all threshold graphs are split and that all split graphs are perfect.

Definition 10 (Split graph). A graph $G$ is called split graph if its nodes can be partitioned into a clique $C$ and a stable set $S$.

The main result of our paper appears in this section as Theorem 15. We prove it with a partitioning technique for the faces of Hansen polytopes of split graphs. We start with describing this partition.

\subsection{Partitioning the faces of Hansen polytopes of split graphs}

Let $G=C \cup S$ be a split graph with clique $C=\left\{c_{1}, \ldots, c_{k}\right\}$ and stable set $S=\left\{s_{1}, \ldots, s_{\ell}\right\}$. A stable set of $G$ is either of the form $A$ or $A \cup\left\{c_{i}\right\}$, for some $A \subseteq S$. Similarly, a clique of $G$ must be either of the form $A$ or $A \cup\left\{s_{j}\right\}$, for some $A \subseteq C$. Thanks to the simple composition of stable sets and cliques of $G$, we can give a complete description of the vertices and facets of $\mathrm{H}(G)$ with the help of Lemma 5. In the following, we omit set parentheses of singletons in order to enhance readability.

- The vertices of $\mathrm{H}(G)$ will be denoted by

(1) $(\varepsilon, A)$ with $\varepsilon= \pm$ and $A \subseteq S$,

(2) $\left(\varepsilon, A \cup c_{i}\right)$ with $\varepsilon= \pm, A \subseteq S$ and $A \cup c_{i}$ stable. 
- The facets of $\mathrm{H}(G)$ will be denoted by

(1) $[\varepsilon, A]$ with $\varepsilon= \pm$ and $A \subseteq C$,

(2) $\left[\varepsilon, A \cup s_{j}\right]$ with $\varepsilon= \pm, A \subseteq C$ and $C \cup s_{j}$ being a clique.

We refer to the different kinds of vertices and facets as type-(1)-vertices/-facets and type(2)-vertices/-facets according to the enumeration above. In the next step, we discuss the vertex-facet incidences. By Lemma 5, a vertex of $\mathrm{H}(G)$ is contained in a facet if and only if they have the same sign and their defining subsets of $V(G)$ intersect, or if they have different signs and the defining subsets are disjoint.

Type-(1)-facets:

- $(\varepsilon, A) \in\left[\varepsilon^{\prime}, B\right] \Longleftrightarrow \varepsilon=-\varepsilon^{\prime}$

- $\left(\varepsilon, A \cup c_{i}\right) \in\left[\varepsilon^{\prime}, B\right] \quad \Longleftrightarrow \quad\left(c_{i} \in B\right.$ and $\left.\varepsilon=\varepsilon^{\prime}\right)$ or $\left(c_{i} \notin B\right.$ and $\left.\varepsilon=-\varepsilon^{\prime}\right)$

Type-(2)-facets:

- $(\varepsilon, A) \in\left[\varepsilon^{\prime}, B \cup s_{j}\right] \quad \Longleftrightarrow \quad\left(s_{j} \in A\right.$ and $\left.\varepsilon=\varepsilon^{\prime}\right)$ or $\left(s_{j} \notin A\right.$ and $\left.\varepsilon=-\varepsilon^{\prime}\right)$

- $\left(\varepsilon, A \cup c_{i}\right) \in\left[\varepsilon^{\prime}, B \cup s_{j}\right] \Longleftrightarrow$ $\left(\varepsilon^{\prime}=\varepsilon\right.$ and $\left(c_{i} \in B\right)$ or $\left.\left(s_{j} \in A\right)\right)$ or $\left(\varepsilon^{\prime}=-\varepsilon\right.$ and $c_{i} \notin B$ and $\left.s_{j} \notin A\right)$

Observe that the events $c_{i} \in B$ and $s_{j} \in A$ are mutually exclusive if $A \cup c_{i}$ is stable and $B \cup s_{j}$ is a clique. The next two lemmas will be of good use later on.

Lemma 11. Let $G=C \cup S$ be a split graph. Choose $A, B \subseteq C$ and $U \subseteq S$ such that $A \cup U$ and $B \cup U$ are cliques. Then,

(i) $[\varepsilon, A \cup U] \cap[\varepsilon, B \cup U]=[\varepsilon,(A \cap B) \cup U] \cap[\varepsilon, A \cup B \cup U]$, and

(ii) $[\varepsilon, A \cup U] \cap[-\varepsilon, B \cup U] \subseteq[\varepsilon, A] \cap[-\varepsilon, B]$.

Proof. The relations can be easily derived by the vertex-facet incidences described before. Let us consider the first claim. In the case that $U$ is the empty set, both intersections $[\varepsilon, A] \cap[\varepsilon, B]$ and $[\varepsilon, A \cap B] \cap[\varepsilon, A \cup B]$ are given by the following set of vertices:

$$
\begin{aligned}
(-\varepsilon, I) \text { with } I \subseteq S, \\
(\varepsilon, I \cup v) \text { with } v \in A \cap B \text { and } I \subseteq S, \\
(-\varepsilon, I \cup v) \text { with } v \notin A \cup B \text { and } I \subseteq S .
\end{aligned}
$$

In the case that, $U=\{i\} \subseteq S$, the two intersections consist of the vertices:

$$
\begin{gathered}
(\varepsilon, I) \text { with } i \in I \subseteq S, \\
(-\varepsilon, I) \text { with } i \notin I \subseteq S, \\
(\varepsilon, I \cup v) \text { with } v \in A \cap B \text { or } i \in I \subseteq S, \\
(-\varepsilon, I \cup v) \text { with } v \notin A \cup B \text { and } i \notin I \subseteq S .
\end{gathered}
$$

Part (ii) follows similarly. 
In particular, part (i) shows that every face can be written using at most two type(1)-facets of each sign. Indeed, for $A_{1}, \ldots, A_{t} \subseteq C$, we get inductively $\bigcap_{i=1}^{t}\left[\varepsilon, A_{i}\right]=$ $\left[\varepsilon, \bigcap_{i=1}^{t} A_{i}\right] \cap\left[\varepsilon, \bigcup_{i=1}^{t} A_{i}\right]$. The next definition relies on this fact and will be essential for the arguments in the upcoming parts.

Definition 12. For a split graph $G$ we define the following four classes of faces of its Hansen polytope $\mathrm{H}(G)$ :

(i) Primitive faces $F$, that are not contained in any type-(1)-facet.

(ii) Positive faces $[+, A] \cap[+, B] \cap F$, with $A \subseteq B$ and $F$ primitive.

(iii) Negative faces $[-, A] \cap[-, B] \cap F$, with $A \subseteq B$ and $F$ primitive.

(iv) Small faces $G$, that are contained in type-(1)-facets of both signs.

This definition gives a partition of the faces of $\mathrm{H}(G)$. For the primitive faces we get a nice characterization with respect to the containment of special vertices.

Lemma 13. Let $G=C \cup S$ be a split graph. A face $F$ of $\mathrm{H}(G)$ is primitive if and only if it contains type-(1)-vertices of both signs.

Proof. $(\Rightarrow)$ Assume $F$ is primitive, i.e., we can write it as

$$
F=\bigcap_{i \in I}\left[+, A_{i} \cup s_{i}\right] \cap \bigcap_{j \in J}\left[-, B_{j} \cup s_{j}\right]
$$

for some multisets $I$ and $J$. If we had $\left\{s_{i}: i \in I\right\} \cap\left\{s_{j}: j \in J\right\} \neq \emptyset$, then Lemma 11 (ii) would yield a contradiction to primitivity. Thus, these two multisets must be disjoint. We get the vertex-facet incidences

- $(+, A) \in F \Longleftrightarrow\left\{s_{i}: i \in I\right\} \subseteq A \subseteq S \backslash\left\{s_{j}: j \in J\right\}$,

- $(-, A) \in F \Longleftrightarrow\left\{s_{j}: j \in J\right\} \subseteq A \subseteq S \backslash\left\{s_{i}: i \in I\right\}$.

This means we can always find positive and negative type-(1)-vertices in $F$.

$(\Leftarrow)$ A vertex $(\varepsilon, A)$ of $F$ cannot be contained in a facet $[\varepsilon, B]$, for every $B \subseteq C$, according to the rules above. So if $F$ contains type-(1)-vertices of both signs, it cannot be contained in any type-(1)-facet. This means that $F$ is primitive.

\subsection{The number of faces of Hansen polytopes of split graphs}

We need the following definition to state our main theorem.

Definition 14. Let $G=C \cup S$ be a split graph. Then, we denote by $p_{G}(C, S)$ the number of partitions of the form $\left(C^{+}, C^{-}, C^{0}, S^{+}, S^{-}, S^{0}\right)$ with $C=C^{+} \cup C^{-} \cup C^{0}$ and $S=S^{+} \cup S^{-} \cup S^{0}$ such that either $C^{+} \cup C^{-} \neq \emptyset$ or $S^{+} \cup S^{-} \neq \emptyset$, and the following hold:

(I) Every element of $C^{+} \cup C^{-}$has a neighbor in $S^{+} \cup S^{-}$.

(II) Every element of $S^{+} \cup S^{-}$has a nonneighbor in $C^{+} \cup C^{-}$. 
In the case of $d$-dimensional Hansen polytopes of split graphs it turns out that $p_{G}(C, S)$ is exactly the number of faces that we have additionally to $3^{d}$. By $\mathrm{s}(P)$ we denote the number of nonempty faces of the polytope $P$.

Theorem 15. Let $G=C \cup S$ be a split graph on $d-1$ nodes. Then

$$
\mathrm{s}(\mathrm{H}(G))=3^{d}+p_{G}(C, S) .
$$

In particular, Hansen polytopes of split graphs satisfy the $3^{d}$ conjecture.

Proof. Let $\Pi$ be the set of all partitions and $\Pi_{A}, \Pi_{B} \subseteq \Pi$ be the subsets for which (I) and (II) hold, respectively. Observe that if (I) fails for a partition, that there must be a node in $C^{+} \cup C^{-}$which is not adjacent to any node in $S^{+} \cup S^{-}$. Thus, this partition fulfills (II). From this we get $\Pi_{A}^{c} \subseteq \Pi_{B}$, where $\Pi_{A}^{c}$ is the complement of $\Pi_{A}$ in $\Pi$. Analogously, $\Pi_{B}^{c} \subseteq \Pi_{A}$ holds. This yields by some simple counting and inclusion-exclusion

$$
3^{d-1}=|\Pi|=\left|\Pi_{A}\right|+\left|\Pi_{B}\right|-\left|\Pi_{A} \cap \Pi_{B}\right| .
$$

Since $p_{G}(C, S)=\left|\Pi_{A} \cap \Pi_{B}\right|-1$, we thus need to show that

$$
\mathrm{s}(\mathrm{H}(G))=3^{d}+\left|\Pi_{A} \cap \Pi_{B}\right|-1=2 \cdot 3^{d-1}+\left|\Pi_{A}\right|+\left|\Pi_{B}\right|-1 .
$$

For this we are going to use the partitioning of the faces of $\mathrm{H}(G)$, that was introduced in Definition 12. Let $f_{p}(G)$ be the number of primitive faces of $\mathrm{H}(G), f_{+}(G)$ be the number of positive, and $f_{-}(G)$ be the number of negative ones. Regarding the small faces, one observes the following: If $F$ is small, then by definition it is contained in type-(1)-facets of both signs. Type-(1)-facets correspond to type-(1)-vertices of the same sign of the polar polytope (via the usual bijection $F \mapsto F^{\star}$ between the face lattice of a polytope and its polar; see $\left[10\right.$, Ch. 2]). Lemma 13 yields that $F^{\star}$ must be a primitive face of $\mathrm{H}(G)^{\star} \cong \mathrm{H}(\bar{G})$. Hence,

$$
\mathrm{s}(\mathrm{H}(G))=f_{p}(G)+f_{+}(G)+f_{-}(G)+f_{p}(\bar{G})-1 .
$$

All we need in order to finish this proof is the following lemma.

Lemma 16. In the setting above we have

(i) $f_{+}(G)=f_{-}(G)=3^{d-1}$, and

(ii) $f_{p}(G)=\left|\Pi_{A}\right|$ and $f_{p}(\bar{G})=\left|\Pi_{B}\right|$.

From this lemma the theorem obviously follows.

Proof of Lemma 16. Recall that $C=\left\{c_{1}, \ldots, c_{k}\right\}$ is the clique and $S=\left\{s_{1}, \ldots, s_{l}\right\}$ is the stable set that $G$ splits into. We need to refine the notion of a primitive face. Given multisets $S^{+}=\left\{s_{i}: i \in I\right\}$ and $S^{-}=\left\{s_{j}: j \in J\right\}$, a primitive face of the form

$$
\bigcap_{i \in I}\left[+, A_{i} \cup s_{i}\right] \cap \bigcap_{j \in J}\left[-, B_{j} \cup s_{j}\right]
$$


is called $\left(S^{+}, S^{-}\right)$-primitive.

(i) The bijection $x \mapsto-x$ maps a facet $[\varepsilon, A]$ of $\mathrm{H}(G)$ to $[-\varepsilon, A]$. Therefore, we have $f_{+}(G)=f_{-}(G)$, and it is enough to show $f_{+}(G)=3^{d-1}$. So let us consider a positive face $P=\left[+, A^{\prime}\right] \cap[+, A] \cap F$, where $A^{\prime} \subseteq A \subseteq C$ and

$$
F=\bigcap_{i \in I}\left[+, A_{i} \cup s_{i}\right] \cap \bigcap_{j \in J}\left[-, B_{j} \cup s_{j}\right]
$$

is primitive. As noted in the proof of Lemma 13, the multisets $\left\{s_{i}: i \in I\right\}$ and $\left\{s_{j}: j \in J\right\}$ are disjoint and $P$ contains a vertex $(-, X), X \subseteq S$, if and only if that vertex is contained in $F$. That is, if and only if $\left\{s_{j}: j \in J\right\} \subseteq X \subseteq S \backslash\left\{s_{i}: i \in I\right\}$. Since there are $3^{|S|}$ many possibilities to choose two disjoint subsets from $S$, it suffices to show that for fixed $\left\{s_{i}: i \in I\right\}$ and $\left\{s_{j}: j \in J\right\}$, we have $3^{|C|}$ many positive faces of the above form.

To this end, let $F$ be a fixed $\left(\left\{s_{i}: i \in I\right\},\left\{s_{j}: j \in J\right\}\right)$-primitive face. The type-(1)vertices of a corresponding positive face $P$ are determined as just explained. Thus it is enough to find out which type-(2)-vertices belong to $P$. We can describe them as

$$
\begin{gathered}
(+, X \cup z) \in P \Longleftrightarrow z \in A^{\prime} \text { and } z \notin \bigcup_{j \in J} B_{j} \text { and } z \in \bigcap_{i \in I, s_{i} \notin X} A_{i} \\
\text { and }\left\{s_{i}: i \in I, z \notin A_{i}\right\} \subseteq X \subseteq S \backslash\left\{s_{j}: j \in J\right\},
\end{gathered}
$$

and similarly

$$
\begin{gathered}
(-, X \cup z) \in P \Longleftrightarrow z \notin A \text { and } z \notin \bigcup_{i \in I} A_{i} \text { and } z \in \bigcap_{j \in J, s_{j} \notin X} B_{j} \\
\text { and }\left\{s_{j}: j \in J, z \notin B_{j}\right\} \subseteq X \subseteq S \backslash\left\{s_{i}: i \in I\right\} .
\end{gathered}
$$

These conditions tell us that, for each $z \in C$, either there is an $X \subseteq S$ such that $(+, X \cup z) \in P$, or there is an $X \subseteq S$ such that $(-, X \cup z) \in P$, or none of these is true. Furthermore, these three cases can be controlled independently by the choices of $A^{\prime}$ and $A$. This gives the desired $3^{|C|}$ positive faces for fixed $\left\{s_{i}: i \in I\right\}$ and $\left\{s_{j}: j \in J\right\}$.

(ii) The second part is a bit more involved. First, as noted before, each partition of $G$ that satisfies (I), automatically satisfies (II) for $\bar{G}$, and vice versa. It is therefore enough to prove $f_{p}(G)=\left|\Pi_{A}\right|$. This is achieved by constructing a bijection $\mathcal{P} \rightarrow \Pi_{A}$, where $\mathcal{P}$ is the set of all primitive faces of $\mathrm{H}(G)$. For this purpose, we partition the domain and range as follows.

Denote by $\mathcal{P}\left(S^{+}, S^{-}\right)$the set of all $\left(S^{+}, S^{-}\right)$-primitive faces. Then

$$
\mathcal{P}=\bigcup\left\{\mathcal{P}\left(S^{+}, S^{-}\right): S^{+}, S^{-} \subseteq S \text { disjoint and } S^{+} \cup S^{-} \neq \emptyset\right\}
$$

is a partition of $\mathcal{P}$.

Let $\Pi_{A}\left(S^{+}, S^{-}\right)$be the set of partitions $\left(C^{+}, C^{-}, C^{0}, S^{+}, S^{-}, S^{0}\right)$ of $V(G)$ that satisfy (I) and where $S^{+}, S^{-}$are fixed (so only $C^{+}, C^{-}$vary). Then

$$
\Pi_{A}=\bigcup\left\{\Pi_{A}\left(S^{+}, S^{-}\right): S^{+}, S^{-} \subseteq S \text { disjoint and } S^{+} \cup S^{-} \neq \emptyset\right\}
$$


is a partition of $\Pi_{A}$.

For the remainder of the proof we let $S^{+}, S^{-}$be fixed and disjoint subsets of $S$ such that $S^{+} \cup S^{-} \neq \emptyset$. We describe mappings

$$
\Psi_{\left(S^{+}, S^{-}\right)}: \mathcal{P}\left(S^{+}, S^{-}\right) \rightarrow \Pi_{A}\left(S^{+}, S^{-}\right)
$$

and

$$
\Phi_{\left(S^{+}, S^{-}\right)}: \Pi_{A}\left(S^{+}, S^{-}\right) \rightarrow \mathcal{P}\left(S^{+}, S^{-}\right),
$$

that will turn out to be inverse to each other. This of course shows that there exists a bijective correspondence between different parts of the partitions of $\mathcal{P}$ and $\Pi_{A}$, which allows us to conclude the existence of a bijection $\mathcal{P} \rightarrow \Pi_{A}$.

Let $\Psi_{\left(S^{+}, S^{-}\right)}$be defined by

$$
\Psi_{\left(S^{+}, S^{-}\right)}(F)=\left(C^{+}, C^{-}, C^{0}, S^{+}, S^{-}, S^{0}\right),
$$

where, for $\varepsilon= \pm$, we let

$$
C^{\varepsilon}=\left\{c \in C:\left(\varepsilon,\left(S^{\varepsilon} \backslash N(c)\right) \cup c\right) \in F \text { and }(-\varepsilon, J \cup c) \notin F, J \subseteq S\right\} .
$$

Here $N(c)$ denotes the neighborhood of $c$ in $G$. On the other hand, define $\Phi_{\left(S^{+}, S^{-}\right)}$by

$$
\Phi_{\left(S^{+}, S^{-}\right)}\left(C^{+}, C^{-}, C^{0}, S^{+}, S^{-}, S^{0}\right)=\bigcap_{s \in S^{+}}\left[+, A_{s}^{\prime} \cup s\right] \cap\left[+, A_{s} \cup s\right] \cap \bigcap_{s \in S^{-}}\left[-, B_{s}^{\prime} \cup s\right] \cap\left[-, B_{s} \cup s\right],
$$

where we let

$$
A_{s}^{\prime}=C^{+} \cap N(s), A_{s}=N(s) \backslash C^{-}, B_{s}^{\prime}=C^{-} \cap N(s) \text { and } B_{s}=N(s) \backslash C^{+} .
$$

We use the abbreviations $\Psi=\Psi_{\left(S^{+}, S^{-}\right)}$and $\Phi=\Phi_{\left(S^{+}, S^{-}\right)}$from now on.

Let us show $\Psi \circ \Phi=\operatorname{id}_{\Pi_{A}\left(S^{+}, S^{-}\right)}$: Given a partition $\pi \in \Pi_{A}\left(S^{+}, S^{-}\right)$it is sufficient to prove

$$
\pi=\left(C^{+}, C^{-}, C^{0}, S^{+}, S^{-}, S^{0}\right) \subseteq \Psi(\Phi(\pi))=\left(D^{+}, D^{-}, D^{0}, S^{+}, S^{-}, S^{0}\right),
$$

where inclusion is to be understood componentwise. Indeed, both $\pi$ and its image are, by construction, partitions of $V(G)$. We begin by explaining $C^{+} \subseteq D^{+}$. Let $c \in C^{+}$. By definition $c \in D^{+}$if the vertex $v=\left(+,\left(S^{+} \backslash N(c)\right) \cup c\right) \in \Phi(\pi)$, and, for all $J \subseteq S$, the vertex $w_{J}=(-, J \cup c) \notin \Phi(\pi)$. Concerning the first condition, we observe that the stable set $\left(S^{+} \backslash N(c)\right) \cup c$ does not hit any of the $B_{s} \cup s$. So $v$ is contained in all of the facets with a negative sign. For the facets with a positive sign the containment is clear if $c \in A_{s}^{\prime}$, and in the case $c \notin A_{s}^{\prime}$, we have $c \notin N(s)$, i.e., $s \in S^{+} \backslash N(c)$. Next consider the second condition on $c$ to be contained in $D^{+}$. Since $\pi$ fulfills (I), there exists a neighbor $s \in S^{+} \cup S^{-}$of $c$. If $s \in S^{+}$, then $c \in C^{+} \cap N(s)=A_{s}^{\prime}$, and therefore $c \in A_{s}^{\prime} \cup s$. This rules out that $(-, J \cup c) \in \Phi(\pi)$. If $s \in S^{-}$, then $c \notin B_{s}^{\prime}$ by construction. So if $w_{J} \in \Phi(\pi)$, we must have $s \in J$ which contradicts the fact that $J \cup c$ is a stable set. Hence $c \in D^{+}$ and thus $C^{+} \subseteq D^{+}$. Similarly, we obtain the inclusion $C^{-} \subseteq D^{-}$. 
It remains to explain $C^{0} \subseteq D^{0}$, so assume $c \in C^{0}$. If $c \notin N\left(S^{+} \cup S^{-}\right)$, then $N(c) \cap S \subseteq$ $S^{0}$ and, in view of the vertex-facet incidences, we get $\left(+, S^{+} \cup c\right),\left(-, S^{-} \cup c\right) \in \Phi(\pi)$, hence $c \in D^{0}$. If $c \in N\left(S^{+} \cup S^{-}\right)$, then there is an $s \in S^{+}$(the case $s \in S^{-}$is analogous) such that $\{c, s\} \in E(G)$. Thus, $c \in C^{0} \cap N(s) \subseteq A_{s}$, meaning that $\left(-,\left(S^{-} \backslash N(c)\right) \cup c\right) \notin \Phi(\pi)$. We also must have $c \notin A_{s}^{\prime}$, from which we get $\left(+,\left(S^{+} \backslash N(c)\right) \cup c\right) \notin \Phi(\pi)$, since $s \in N(c)$. This shows $c \in D^{0}$, and concludes $\Psi \circ \Phi=\operatorname{id}_{\Pi_{A}\left(S^{+}, S^{-}\right)}$.

Now we show $\Phi \circ \Psi=\operatorname{id}_{\mathcal{P}\left(S^{+}, S^{-}\right)}$: Given an $\left(S^{+}, S^{-}\right)$-primitive face

$$
F=\bigcap_{s \in S^{+}}\left[+, A_{s}^{\prime} \cup s\right] \cap\left[+, A_{s} \cup s\right] \cap \bigcap_{s \in S^{-}}\left[-, B_{s}^{\prime} \cup s\right] \cap\left[-, B_{s} \cup s\right],
$$

we need to show $\Phi(\Psi(F))=F$. Both $F$ and its image are $\left(S^{+}, S^{-}\right)$-primitive faces and hence they contain type-(1)-vertices $(\varepsilon, J)$ if and only if $S^{\varepsilon} \subseteq J \subseteq S \backslash S^{-\varepsilon}$. Therefore, we only need to show that both $F$ and $\Phi(\Psi(F))$ contain the same type-(2)-vertices as well.

We begin with showing that $(\varepsilon, J \cup c) \in F$ implies $(\varepsilon, J \cup c) \in \Phi(\Psi(F))$. To this end, we let $(\varepsilon, J \cup c) \in F$ and we distinguish two cases.

1) Assume there exists a $K \subseteq S$ such that $(-\varepsilon, K \cup c) \in F$. This means, that $c$ cannot be contained in $A_{s}$ or $B_{s}$, for $s \in S^{+}$or $s \in S^{-}$, respectively. Due to $(\varepsilon, J \cup c) \in F$, we must have $S^{-\varepsilon} \subseteq K$ and $S^{\varepsilon} \subseteq J \subseteq S \backslash S^{-\varepsilon}$. From here we see that $c$ has no neighbor in $S^{-\varepsilon}$. Altogether, this yields $(\varepsilon, J \cup c) \in\left[-\varepsilon,\left(C^{-\varepsilon} \cap N(s)\right) \cup s\right]$, for all $s \in S^{-\varepsilon}$, and $(\varepsilon, J \cup c) \in\left[\varepsilon,\left(C^{\varepsilon} \cap N(s)\right) \cup s\right]$, for all $s \in S^{\varepsilon}$. Hence, $(\varepsilon, J \cup c) \in \Phi(\Psi(F))$ as desired.

2) The other case is $(-\varepsilon, K \cup c) \notin F$, for all $K \subseteq S$. If $s \in S^{\varepsilon}$ is not adjacent to $c$, then $s \in J$, i.e., $S^{\varepsilon} \backslash N(c) \subseteq J$. According to (1) and $(\varepsilon, J \cup c) \in F$, we also have $c \in C^{\varepsilon}$, independently of $s$. So, for any $s \in S^{\varepsilon}$, either $s \in J$ or $c \in C^{\varepsilon} \cap N(s)$. From this we get that $(\varepsilon, J \cup c)$ is contained in every facet of sign $\varepsilon$ that defines $\Phi(\Psi(F))$. Since $J \cap S^{-\varepsilon}=\emptyset$, we conclude that $(\varepsilon, J \cup c)$ is also contained in every facet of sign $-\varepsilon$. Again we obtain $(\varepsilon, J \cup c) \in \Phi(\Psi(F))$.

We finally need to prove also the converse direction, that is, $(\varepsilon, J \cup c) \in \Phi(\Psi(F))$ implies $(\varepsilon, J \cup c) \in F$. By the vertex-facet incidences, we have $J \subseteq S \backslash S^{-\varepsilon}$, for all $(\varepsilon, J \cup c) \in \Phi(\Psi(F))$. Again, we distinguish between two cases.

1) Let $S^{\varepsilon} \subseteq J$. If $c \notin N\left(S^{-\varepsilon}\right)$, then, by $S^{\varepsilon} \subseteq J,(\varepsilon, J \cup c)$ is contained in all facets with sign $\varepsilon$ that define $F$. Since the cliques corresponding to the facets with sign $-\varepsilon$ contain only nodes from $N\left(S^{-\varepsilon}\right)$ or $S^{-\varepsilon}$, and $J \subseteq S \backslash S^{-\varepsilon}$, the vertex $(\varepsilon, J \cup c)$ lies in all facets defining $F$. So, now let $c \in N\left(S^{-\varepsilon}\right)$. By $(\varepsilon, J \cup c) \in \Phi(\Psi(F))$, we have $(\varepsilon, J \cup c) \in$ $\left[-\varepsilon,\left(N(s) \backslash D^{\varepsilon}\right) \cup s\right]$, for all $s \in S^{-\varepsilon}$, where $D^{\varepsilon}$ is a component of $\Psi(F)$. Therefore, $c \notin N(s) \backslash D^{\varepsilon}$, for all $s \in S^{-\varepsilon}$, and thus $c \in D^{\varepsilon}$. This implies that $\left(\varepsilon,\left(S^{\varepsilon} \backslash N(c)\right) \cup c\right) \in F$, which gives $(\varepsilon, J \cup c) \in F$ because $S^{\varepsilon} \subseteq J \subseteq S \backslash S^{-\varepsilon}$.

2) On the other hand, consider $S^{\varepsilon} \nsubseteq J$. Then, because of $(\varepsilon, J \cup c) \in \Phi(\Psi(F))$, we have $c \in D^{\varepsilon} \cap N(s)$, for all $s \in S^{\varepsilon} \backslash J$, where $D^{\varepsilon}$ is again a component of $\Psi(F)$. In particular, $c \in D^{\varepsilon}$, which means that the vertex $\left(\varepsilon,\left(S^{\varepsilon} \backslash N(c)\right) \cup c\right) \in F$. In view of $J \subseteq S \backslash S^{-\varepsilon}$ and $c \in N(s)$, for any $s \in S^{\varepsilon} \backslash J$, this implies $(\varepsilon, J \cup c) \in F$.

This finishes the argument for $\Psi \circ \Phi=\operatorname{id}_{\Pi_{A}\left(S^{+}, S^{-}\right)}$, and therefore establishes the bijection $\mathcal{P} \rightarrow \Pi_{A}$. 
In particular, Theorem 15 says that the partition of the split graph does not play any role in the number of vertices of the corresponding Hansen polytope. So instead of $p_{G}(C, S)$ we write $p_{G}$ from now on. What we know about this function is summarized by the following corollary.

Corollary 17. Let $G=C \cup S$ be a split graph on $d-1$ nodes. Then

$$
\mathrm{s}(\mathrm{H}(G))=3^{d}+16 \cdot \ell, \quad \text { for some } \ell \in \mathbb{N},
$$

with $\ell=0$ if and only if $G$ is a threshold graph.

Proof. Let us first establish that $p_{G}=16 \cdot \ell$. Assume that $C=C^{+} \bullet C^{-} \bullet C^{0}$ and $S=S^{+} \cup S^{-} \bullet S^{0}$ is given. If $C^{+} \cup C^{-}=\emptyset$, then (II) is only satisfied if $S^{+} \cup S^{-}=\emptyset$. Similarly, if $S^{+} \cup S^{-}=\emptyset$, we have $C^{+} \cup C^{-}=\emptyset$ because of (I). In both cases we deal with the trivial partition $C^{0}=C, S^{0}=S$ that is not counted by $p_{G}$, and thus can be ignored. If $C^{+} \cup C^{-}=\{c\}$, then by (I) there exists a neighbor of $c$ in $S^{+} \cup S^{-}$. By (II) again, this neighbor must have a nonneighbor in $C^{+} \cup C^{-}$, which clearly cannot be. So also this case is not counted by $p_{G}$ and can be ignored as well. By similar reasoning, we can disregard the case $S^{+} \cup S^{-}=\{s\}$. Therefore, we must have $\left|C^{+} \cup C^{-}\right| \geqslant 2$ and $\left|S^{+} \cup S^{-}\right| \geqslant 2$. Since we can assign the elements to $C^{+}, C^{-}$or $S^{+}, S^{-}$in an arbitrary way, we get that $p_{G}=16 \cdot \ell$, for some $\ell \in \mathbb{N}$.

Now $\ell=0$ if and only if $p_{G}=0$. If $G$ has a path on four nodes $P_{4}$ as an induced subgraph, then the partition where $C^{+}$is the two middle nodes of $P_{4}, S^{+}$is the two endpoints and $C^{-}=S^{-}=\emptyset$, satisfies the conditions (I) and (II). So if $\ell=0$, then $G$ is a split graph with no induced path of four nodes. By [6, Thm. 1.2.4], this implies that $G$ is a threshold graph. On the other hand, if $G$ is threshold then $\mathrm{H}(G)$ is a Hanner polytope by Theorem 8 , hence $\ell=0$.

\subsection{High-dimensional Hansen polytopes with few faces}

In the rest of this section, we study a construction that leads us to high-dimensional Hansen polytopes with few faces. To this end, consider a threshold graph $T$ on $m$ nodes and a split graph $G=C \cup S$ on $n$ nodes. We construct a new graph $G \ltimes T$ by taking the union of $G$ and $T$ and adding edges between every node of the clique $C$ of $G$ and every node of $T$. Figure 1 is an illustration of our construction with $G$ being the path on four nodes. It is clear that the resulting graph is again a split graph and therefore perfect.

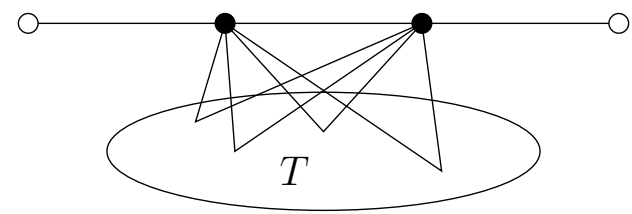

Figure 1: Appending a threshold graph to a split graph. 
Proposition 18. Let $G=C \cup S$ be a split graph on $n$ nodes. Then, for any threshold graph $T$ on $m$ nodes, we have

$$
\mathrm{s}(\mathrm{H}(G \ltimes T))=3^{m+n+1}+p_{G} .
$$

This means $p_{G \ltimes T}=p_{G}$, and therefore $p_{G \ltimes T}$ is independent of $T$.

Proof. By definition the threshold graph $T$ can be built up by successively adding isolated and dominating nodes. This induces an ordering on the nodes $v_{1}, \ldots, v_{m}$ of $T$. Let $C_{T}:=\left\{v_{i}: v_{i}\right.$ dominating at step $\left.i\right\}$ and $S_{T}:=\left\{v_{i}: v_{i}\right.$ isolated at step $\left.i\right\}$. This splits $T$ into a clique $C_{T}$ and a stable set $S_{T}$, which in turn splits $G \ltimes T$ into $C \cup C_{T}$ and $S \cup S_{T}$. By construction every node in $C_{T}$ and $S_{T}$ is connected to all nodes in $C$ and none in $S$. Now consider a partition $\left(C^{+} \cup C^{-} \cup C^{0}, S^{+} \cup S^{-} \cup S^{0}\right)$ of $G \ltimes T$ that is counted by $p_{G \ltimes T}\left(C \cup C_{T}, S \cup S_{T}\right)$. By (I) for all $x \in\left(C^{+} \cup C^{-}\right) \cap C_{T}$ there exists a neighbor $y \in\left(S^{+} \cup S^{-}\right) \cap S_{T}$, which means that in $T$ the once isolated node $y$ was inserted before the once dominating node $x$. On the other hand, by (II), any given node $y \in\left(S^{+} \cup S^{-}\right) \cap S_{T}$ has to have a nonneighbor $z \in\left(C^{+} \cup C^{-}\right) \cap C_{T}$. Such a node $z$ was used before $y$ in the construction of $T$. These two observations can only hold in the case $\left(C^{+} \cup C^{-}\right) \cap C_{T}=\emptyset=\left(S^{+} \cup S^{-}\right) \cap S_{T}$. Therefore, for this partition we have $C_{T} \subseteq C^{0}$ and $S_{T} \subseteq S^{0}$, which implies that $p_{G \ltimes T}\left(C \cup C_{T}, S \cup S_{T}\right)=p_{G}$.

This finally yields a series of high-dimensional Hansen polytopes with very few faces.

Corollary 19. Let $P_{4}$ be a path on four nodes and let $T$ be an arbitrary threshold graph on $m$ nodes. Then

$$
\mathrm{s}\left(\mathrm{H}\left(P_{4} \ltimes T\right)\right)=3^{m+5}+16 .
$$

Proof. Determining $p_{P_{4} \ltimes T}=p_{P_{4}}=16$ is an easy counting exercise.

\section{Experimental results on related conjectures}

Our experimental evidence indicates that the polytopes constructed in Corollary 19 are of interest also in view of two further minimization problems where it is also conjectured that the Hanner polytopes yield the only optimal examples. The first one is a question by Kalai about the minimal number of full flags of a centrally symmetric $d$-polytope; the second one is the classical Mahler conjecture from 1939 about the minimal product of volumes of a centrally symmetric polytope and its polar dual.

To make this concrete, define the full flags of a $d$-dimensional polytope $P$ as the chains $\emptyset \subset F_{0} \subset F_{1} \subset \ldots \subset F_{d-1} \subset P$, where each $F_{i}$ is an $i$-dimensional face of $P$. We write $\mathrm{ff}(P)$ for the number of full flags of $P$. (This parameter $\mathrm{ff}(P)$ also counts the number of maximal faces of a barycentric subdivision of $P$, which in special situations, as in the case of unimodular barycentric subdivisions, might be related to the volumes of $P$ and its polar dual.) 
Conjecture 20 (Kalai). Let $P \subset \mathbb{R}^{d}$ be a centrally symmetric polytope and let $H$ be a $d$-dimensional Hanner polytope. Then

$$
\mathrm{ff}(P) \geqslant \mathrm{ff}(H)=2^{d} d !
$$

where equality holds only for Hanner polytopes.

For Mahler's conjecture, recall that $P^{\star}=\left\{x \in \mathbb{R}^{d}:\langle x, y\rangle \leqslant 1\right.$ for all $\left.y \in P\right\}$ defines the polar polytope of $P$ and denote the volume in $\mathbb{R}^{d}$ by $\operatorname{vol}(\cdot)$.

Conjecture 21 (Mahler [7]). Let $P \subset \mathbb{R}^{d}$ be a centrally symmetric polytope and let $H$ be a $d$-dimensional Hanner polytope. Then

$$
\operatorname{vol}(P) \cdot \operatorname{vol}\left(P^{\star}\right) \geqslant \operatorname{vol}(H) \cdot \operatorname{vol}\left(H^{\star}\right)=\frac{4^{d}}{d !},
$$

where equality holds only for Hanner polytopes.

Now let $G_{d}=P_{4} \ltimes T$ be an instance of Corollary 19, where $T$ is some threshold graph on $d-5$ nodes. The corresponding Hansen polytope $\mathrm{H}\left(G_{d}\right)$ is $d$-dimensional. A complete computer enumeration for all such graphs $G_{d}$ showed that for $d \leqslant 10$ the numbers of full flags and the Mahler volume product are again independent of the actual choice of $T$ :

$$
\mathrm{ff}\left(\mathrm{H}\left(G_{d}\right)\right)=2^{d} d !\left(1+4 \frac{(d-4) !}{d !}\right)
$$

and

$$
\operatorname{vol}\left(\mathrm{H}\left(G_{d}\right)\right) \cdot \operatorname{vol}\left(\mathrm{H}\left(G_{d}\right)^{\star}\right)=\frac{4^{d}}{d !}\left(1+\frac{(d-4) !}{d !}\right)
$$

We expect that these formulas hold for all dimensions $d \geqslant 5$, so that the graphs $G_{d}$ lead to centrally symmetric polytopes that are simultaneously very close to the conjectured lower bounds in the $3^{d}$ conjecture, the full flag conjecture, and the Mahler conjecture. This supports the impression that there is a close relation between, in particular, Conjectures 20 and 21.

\section{Acknowledgements}

This work has been started while the first two authors enjoyed the hospitality of the Centre de Recerca Matemàtica in Barcelona. We are grateful to Michael Joswig and Eugen Gawrilow for the development of polymake [2]: Without this software a considerable part of our work would not have been possible. 


\section{References}

[1] Reinhard Diestel: Graph Theory, Graduate Texts in Math. 173, 4th ed., Springer New York, 2010.

[2] Ewgenij Gawrilow and Michael Joswig: polymake: a framework for analyzing convex polytopes. In: Polytopes - Combinatorics and Computation (G. Kalai and G. M. Ziegler, eds.), Birkhäuser, Basel 2000, pp. 43-74.

[3] Olof Hanner: Intersections of translates of convex bodies. Mathematica Scandinavica, 4:67-89, 1956.

[4] Allan B. Hansen: On a certain class of polytopes associated with independence systems. Mathematica Scandinavica, 41:225-241, 1977.

[5] Gil Kalai: The number of faces of centrally-symmetric polytopes. Graphs and Combinatorics, 5:389-391, 1989.

[6] Nadimpalli V. R. Mahadev and Uri N. Peled: Threshold Graphs and Related Topics, Annals of Discrete Math. 56, North-Holland, 1995.

[7] Kurt Mahler: Ein Übertragungsprinzip für konvexe Körper. Československá Akademie Věd. Časopis Pro Pěstováni Matematiky, 68:93-102, 1939.

[8] Raman Sanyal, Axel Werner, and Günter M. Ziegler: On Kalai's conjectures concerning centrally symmetric polytopes. Discrete Comput. Geometry, 41:183-198, 2009.

[9] Alexander Schrijver: Combinatorial Optimization, Vol. B, Algorithms and Combinatorics 24, Springer, Heidelberg 2003.

[10] Günter M. Ziegler: Lectures on Polytopes, Graduate Texts in Mathematics 152, Springer, New York 1995. 